Pacific Journal of Mathematics

FUNCTIONALLY COMPACT SPACES, $C$-COMPACT SPACES
AND MAPPINGS OF MINIMAL HAUSDORFF SPACES 


\title{
FUNCTIONALLY COMPACT SPACES, C-COMPACT SPACES AND MAPPINGS OF MINIMAL HAUSDORFF SPACES
}

\author{
S. W. WILLARD
}

Our interest in this paper is in the mapping properties of minimal Hausdorff spaces; some of the results will provide new characterizations of the classes of functionally compact and $C$-compact spaces. Of more than secondary interest, it may be the primary message of the paper, is the point of view adopted (and outlined in $\$ 2$ ) in studying the "divisibility" of the highly nondivisible class of minimal Hausdorff spaces.

1. Introduction. Let $X$ be a Hausdorff space. Then $X$ is absolutely closed (AC) iff whenever $X$ is embedded in a Hausdorff space $Y, X$ is closed in $Y$. We call $X$ minimal Hausdorff (MH) iff $X$ admits no one-to-one continuous map to a Hausdorff space which is not a homeomorphism. $X$ is functionally compact (FC) iff every continuous map on $X$ to a Hausdorff space is a closed map. Finally, Velicko [13] has defined a set $A$ in a space $X$ to be an $H$-set iff for each family of sets open in $X$ and covering $A$, there is a finite subfamily whose closures in $X$ cover $A$. Porter and Thomas [11; Thm. 2.5] have observed that in Hausdorff spaces $H$-sets are closed, and Viglino [14] has defined a Hausdorff space to be $C$-compact $(C C)$ iff every closed set is an $H$-set.

Some of the basic results we will need concerning the classes of spaces defined above are given in the following theorem.

THEOREM 1.1. Let $X$ be a Hausdorff space. Then

(a) ([4]) $X$ is $A C$ iff every open filter on $X$ has a cluster point,

(b) ([4]) $X$ is $\mathrm{MH}$ iff every open filter on $X$ with a unique cluster point converges (necessarily to that point),

(c) ([5]) $X$ is FC iff whenever $\mathscr{U}$ is an open filter base on $X$ such that $\cap\{U \mid U \in \mathscr{Q}\}=\cap\{\bar{U} \mid U \in \mathscr{Q}\}$, then $\mathscr{C}$ is a base for the neighborhoods of $\cap\{\bar{U} \mid U \in \mathscr{Q}\}$.

(d) ([15]) $X$ is CC iff every open filter base $\mathscr{Q}$ on $X$ is a base for the nhoods of $\cap\{\bar{U} \mid U \in \mathscr{Q}\}$.

Each of the characteristic properties above can be applied to non-Hausdorff spaces. For example, a (not necessarily Hausdorff) space $X$ is generalized minimal Hausdorff (GMH) iff every open filter with a unique cluster point converges. Similar definitions can be given for generalized absolutely closed (GAC), generalized functionally compact (GFC) and generalized C-compact (GCC) spaces. 
The following theorem displays the relationships between the properties introduced so far.

THEOREM 1.2. Compact $\Rightarrow \mathrm{CC} \Rightarrow \mathrm{FC} \Rightarrow \mathrm{MH} \Rightarrow \mathrm{AC}$ and none of these implications can, in general, be reversed.

The proof, as well as the necessary counterexamples can be found divided between [14], [5], [7], [12] and [4].

2. Mappings of minimal Hausdorff spaces. Products and continuous images of compact spaces are compact; it has been a continuing object of interest in investigations concerning the weaker versions of compactness introduced above to discover the extent to which these properties are similarly productive and divisible. It will be convenient to introduce, at this point, the term Hausdorff divisible, which will designate those properties of topological spaces which are preserved by quotient maps with Hausdorff range. Our investigation will center on the study of Hausdorff divisibility in the class of $\mathrm{MH}$ spaces.

This class is not Hausdorff divisible. In fact, the stock example of a non- $\mathrm{MH} \mathrm{AC}$ space is a perfect (= closed, continuous with compact point-inverses) image of the stock example of a non-compact $\mathrm{MH}$ space (see [3]). Whenever, as here, a class $\mathscr{P}$ of topological spaces is badly treated by a class $\mathscr{L}$ of maps, a great deal of information can be derived by considering two related classes:

$R_{\mathscr{Z}}(\mathscr{P})$ : the class of spaces whose every $\mathscr{L}$-image lies in $\mathscr{P}$, and $P(\mathscr{P})$ : the class of spaces which are $\mathscr{L}$-images of spaces from class $\mathscr{P}$.

Note that these classes are, respectively, the largest class smaller than $\mathscr{P}$ which is closed under $\mathscr{L}$-maps and the smallest class larger than $\mathscr{P}$ which is closed under $\mathscr{L}$-maps, assuming that the class $\mathscr{L}$ includes all identity maps. These facts make $R(\mathscr{P})$ and $P(\mathscr{P})$ natural objects for study whenever the class $\mathscr{P}$ is not itself closed under $\mathscr{L}$-maps.

Arhangelskii [1] specifically identified $P(\mathscr{P})$ as an object of concern, but failed to mention $R_{\mathscr{P}}(\mathscr{P})$. In this section, we will determine $R, \mathscr{P})$ for the class $\mathscr{P}$ of $\mathrm{MH}$ spaces and the class $\mathscr{L}$ of continuous maps whose domain and range are Hausdorff (Theorem 2.1), use this to prove a rather curious corollary (2.3) and, along the way, provide new characterizations of the class of FC spaces (2.1).

THEOREM 2.1. The following are equivalent, for a Hausdorff space $X$ : 
(a) $X$ is $\mathrm{FC}$,

(b) every continuous map of $X$ onto a Hausdorff space is a quotient map,

(c) every continuous Hausdorff image of $X$ is $\mathrm{MH}$,

(d) every Hausdorff quotient of $X$ is $\mathrm{MH}$,

(e) every closed continuous Hausdorff image of $X$ is $\mathrm{MH}$.

Proof. $\quad(\mathrm{a}) \Rightarrow$ (b) and $(\mathrm{c}) \Rightarrow(\mathrm{d}) \Rightarrow$ (e) are obvious.

(b) $\Rightarrow(\mathrm{c})$ : Suppose $X$ has the property of (b) and $f$ is a continuous map of $X$ onto a Hausdorff space $Y$. If $Y$ is not minimal Hausdorff, let $Y^{*}$ be the set $Y$ with a strictly weaker Hausdorff topology. Then $f$ defines a map $f^{*}: X \rightarrow Y^{*}$ which is continuous but cannot be a quotient mapping, a contradiction.

(e) $\Rightarrow$ (a): Suppose $X$ is not FC. Then, by Theorem 1.1, for some open filter base $\mathscr{C}$ on $X, \cap\{U \mid U \in \mathscr{Q}\}=\cap\{\bar{U} \mid U \in \mathscr{C}\}=A$, while $\mathscr{C}$ is not a neighborhood base at $A$. Now if $A$ is empty, $X$ itself is not $\mathrm{MH}$, which is impossible. On the other hand, if $A$ is nonempty, then the quotient $Z$ obtained from $X$ by identifying the points of $A$ is Hausdorff, but not minimal Hausdorff. For it can be retopologized as a Hausdorff space with a strictly smaller neighborhood base at $A$. Since the quotient map of $X$ onto $Z$ is closed, we are done.

Part (b) of the last theorem makes it clear that the study of the class of FC spaces is the study of what, at first glance, would seem to be a wider and more natural class of spaces, i.e., those spaces $X$ with the property that, if $f$ is a continuous map from $X$ to a Hausdorff space $Y$, then $f$ is a quotient map.

Theorem 2.1 also has an obvious, but rather curious, consequence (Theorem 2.3), for which we require the following result.

THEOREM 2.2. Let $X$ be a topological space. If the projection $X \times Y \rightarrow Y$ is closed for each compact Hausdorff space $Y$, then $X$ is compact.

Proof. Let $n$ be an infinite cardinal, $\Omega_{n}$ the least ordinal of cardinal $n$. According to a result of Noble [10; Thm. 2.2], if $Y$ contains a point $y$ such that

(a) $\boldsymbol{n}$ is the smallest cardinal of a neighborhood base at $y$, and

(b) there is a family $\left\{S_{\alpha} \mid \alpha \in \Omega_{n}\right\}$ of closed subsets of $Y$ such that $y$ is in the closure of $\bigcup_{\alpha \in \Omega_{n}} S_{\alpha}$, but not in the closure of $\bigcup_{\alpha<\alpha_{0}} S_{\alpha}$ for any $\alpha_{0} \in \Omega_{n}$,

then for the projection $\pi_{x}: X \times Y \rightarrow Y$ to be closed, it is necessary that every open cover of $X$ of cardinarity $n$ has a subcover of cardinality $<\boldsymbol{n}$.

Since compact Hausdorff spaces $Y$ can be found satisfying con- 
ditions (a) and (b) above for any infinite cardinal $\boldsymbol{n}$ (for example, $Y=$ the one-point compactification of $\Omega_{n}$ ), we conclude that every infinite open cover of $X$ has a subcover of strictly smaller cardinality, whence (easily) $X$ is compact.

Theorem 2.3. (a) If a class $\mathscr{P}$ of spaces contained in the class of FC spaces and containing the class of compact Hausdorff spaces is productive, then $\mathscr{P}$ is the class of compact Hausdorff spaces.

(b) If a class $\mathscr{P}$ of spaces contained in the class of $\mathrm{MH}$ spaces and containing the class of compact Hausdorff spaces is productive and Hausdorff divisible, then $\mathscr{P}$ is the class of compact Hausdorff spaces.

(c) If a noncompact space $X$ is $\mathrm{FC}$ or $\mathrm{CC}$, then there is a compact Hausdorff space $Y$ such that $X \times Y$ is not FC.

Proof. (a) and (c) follow directly from Lemma 2.2; (b) follows from 2.1 and (a).

Thus "nice" properties between compactness and the MH property are confined to one, compactness itself. This use of Theorem 2.1 is a good example of the utility of the concepts $R_{\mathscr{L}}(\mathscr{P})$ and $P_{\mathscr{C}}(\mathscr{P})$ for nondivisible classes.

The problem of determining $P_{\mathscr{C}}(\mathscr{P})$ for the class $\mathscr{P}$ of $\mathrm{MH}$ spaces and the class $\mathscr{L}$ of maps with Hausdorff range remains open. The following is an attractive conjecture:

Conjecture. A Hausdorff space $X$ is AC iff it is the continuous image of some $\mathrm{MH}$ space.

3. C-compact Spaces. By relaxing the separation axiom in 2.1 (c), (d), and (e), one obtains a characterization of $C$-compact spaces and determines $R_{\mathscr{C}}(\mathscr{P})$ for the class $\mathscr{P}$ of GMH spaces and the class $\mathscr{L}$ of continuous maps with Hausdorff domain and $T_{1}$ range. To introduce this, we give a preliminary characterization of GCC spaces. We will use the following terminology: an open filter base $\mathscr{C}$ on a topological space $X$ converges to a set $A \subseteq X$ iff every nhood $V$ of $A$ contains an element of $\mathscr{C}$, and an open filter base $\mathscr{C}$ meets a set $B \subseteq X$ iff $U \cap B \neq 0$ for each $U \in \mathscr{Q}$.

Lemma 3.1. The following are equivalent for a space $X$ :

(a) $X$ is GCC,

(b) every closed set in $X$ is an $H$-set,

(c) the continuous image of $X$ is GCC, and

(d) if $A$ is closed in $X$ and $\mathscr{Q}$ is an open filter base which meets $A$, then $\mathscr{U}$ has a cluster point in $A$. 
Proof. The proof is straightforward.

THEOREM 3.2. The following are equivalent for a Hausdorff space $X$ :

(a) $X$ is C-compact,

(b) every continuous $T_{1}$ image of $X$ is $\mathrm{GMH}$,

(c) every $T_{1}$ quotient of $X$ is GMH, and

(d) every closed continuous $T_{1}$ image of $X$ is GMH.

Proof. Clearly (b) implies (c) and (c) implies (d). That (a) implies (b) follows immediately from 3.1 and 1.1. To prove (d) implies (a), suppose $X$ is not $C$-compact. Then, say, $\mathscr{C}$ is an open filter base in $X$ with $C=\cap\{\bar{U} \mid U \in \mathscr{Q}\}$, while $\mathscr{Q}$ does not converge to $C$, by 3.1. Obtain a quotient $Z$ of $X$ by identifying the points of $C$; call the quotient map $h$. Then $Z$ is $T_{1}$ and $h$ is a closed continuous map of $X$ onto $Z$, but $Z$ is not GMH. For, by an easy rearrangement, we may assume $C$ has no interior and $U \cap C=\varnothing$ for each $U \in \mathscr{U}$. Now if $U \in \mathscr{Q}$, then $h(U)$ is open in $Z$ and each nhood of $h(C)=p$ meets $h(U)$, so $p \in \overline{h(U)}$. Moreover, if $q \neq p$ in $Z$, then some nhood of $q$ fails to meet some $h(U)$ [else $h^{-1}(q)$ be a point in $\cap \bar{U}$ which is not in $C$ ], so $\cap \overline{h(U)}=\{p\}$. But if $V$ is a nhood of $C$ in $X$ which contains no $U \in \mathscr{U}$, then $h(V)$ is a nhood of $p$ which contains no $h(U)$. Thus we have an open filter base $\{h(U) \mid U \in \mathscr{Q}\}$ in $Z$ with a unique cluster point $p$ which does not converge to $p$. So $Z$ is not GMH.

\section{REFERENCES}

1. Al Arhangel'skii, Mappings and spaces, Russian Math. Surveys, 21 No. 4 (1966), 115-162.

2. B. Banaschewski, Ueber Hausdorffsche-minimale Erweiterung von Raumen, Arch. Math., 12 (1961), 355-365.

3. M. Berri, Minimal topological spaces, Trans. Amer. Math. Soc., 108 (1963), 97-105.

4. N. Bourbaki, Topologie generale, Act. Scie. Ind., 858-1142, Hermann, Paris, 1951.

5. R. F. Dickman, Jr. and A. Zame, Functionally Compact Spaces, Pacific J. Math., 31 (1970), 303-311.

6. - Every Hausdorff space can be embedded in a Hausdorff space on which every mapping is closed, Notices Amer. Math. Soc., 17 (1970), 466.

7. G. K. Goss, and G. A. Viglino, C-compact and functionally compact spaces, Notices Amer. Math. Soc., 17, no. 2, (February (1970), p. 468.

8. M. Katetov, Ueber H-abgeschlossene und bikompakte Raeume, Casopis Pro. Pest. Mat. a Fys., 69 (1940), 36-49.

9. Chen Tung Liu, Absolutely closed spaces, Trans Amer. Math. Soc., 130 (1968), 86-104.

10. N. Noble, Products with closed projections, Trans. Amer. Math. Soc., 140 (1969), 381-391.

11. J. Porter, and J. Thomas, On H-closed and minimal Hausdorff spaces, Trans. Amer. Math. Soc., 138 (1969), 159-170. 
12. C. T. Scarborough, and A. H. Stone, Products of nearly compact spaces, Trans. Amer. Math. Soc., 124 (1966), 131-147.

13. N. V. Velicko, H-closed topological spaces, Mat. Sb., 70 (112) (1966), 98-112= Amer. Math. Soc. Transl., 78 (2) (1968), 103-118.

14. Giovanni Viglino, C-compact spaces, Duke Math. J., 36 (1969), 761-764.

15. - Seminormal and C-compact spaces, to appear.

Received June 16, 1970 and in revised form September 29, 1970. The author was supported by NCR Grant A-7591.

University of Alberta, Canada 


\section{PACIFIC JOURNAL OF MATHEMATICS}

\section{EDITORS}

H. SAMELSON

Stanford University

Stanford, California 94305

C. R. Hовву

University of Washington

Seattle, Washington 98105
J. DUGUNDJI

Department of Mathematics

University of Southern California

Los Angeles, California 90007

RICHARD ARENS

University of California

Los Angeles, California 90024

\section{ASSOCIATE EDITORS}
E. F. BECKENBACH
B. H. NeumanN
F. WOLF
K. YoSHIDA

\section{SUPPORTING INSTITUTIONS}

UNIVERSITY OF BRITISH COLUMBIA

CALIFORNIA INSTITUTE OF TECHNOLOGY

UNIVERSITY OF CALIFORNIA

MONTANA STATE UNIVERSITY

UNIVERSITY OF NEVADA

NEW MEXICO STATE UNIVERSITY

OREGON STATE UNIVERSITY

UNIVERSITY OF OREGON

OSAKA UNIVERSITY

UNIVERSITY OF SOUTHERN CALIFORNIA
STANFORD UNIVERSITY

UNIVERSITY OF TOKYO

UNIVERSITY OF UTAH

WASHINGTON STATE UNIVERSITY

UNIVERSITY OF WASHINGTON

AMERICAN MATHEMATICAL SOCIETY CHEVRON RESEARCH CORPORATION NAVAL WEAPONS CENTER 


\section{Pacific Journal of Mathematics}

\section{Vol. 38, No. $1 \quad$ March, 1971}

Bruce Alan Barnes, Banach algebras which are ideals in a Banach algebra ..... 1

David W. Boyd, Inequalities for positive integral operators............... 9

Lawrence Gerald Brown, Note on the open mapping theorem .............. 25

Stephen Daniel Comer, Representations by algebras of sections over Boolean

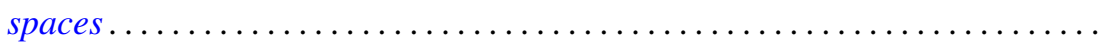

John R. Edwards and Stanley G. Wayment, On the nonequivalence of

conservative Hausdorff methods and Hausdorff moment sequences ........

P. D. T. A. Elliott, On the limiting distribution of additive functions $(\bmod 1) \ldots \ldots$

Mary Rodriguez Embry, Classifying special operators by means of subsets

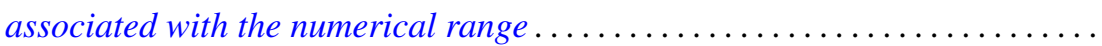

Darald Joe Hartfiel, Counterexamples to a conjecture of G. N. de Oliveira ......

C. Ward Henson, A family of countable homogeneous graphs...............

Satoru Igari and Shigehiko Kuratsubo, A sufficient condition for

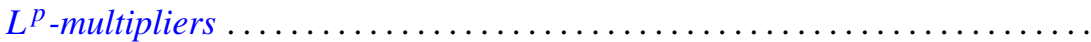

William A. Kirk, Fixed point theorems for nonlinear nonexpansive and

generalized contraction mappings............................

Erwin Kleinfeld, A generalization of commutative and associative rings ...... 95

D. B. Lahiri, Some restricted partition functions. Congruences modulo $11 \ldots \ldots 103$

T. Y. Lin, Homological algebra of stable homotopy ring $\pi *$ of spheres ....... 117

Morris Marden, A representation for the logarithmic derivative of a meromorphic function...........................

John Charles Nichols and James C. Smith, Examples concerning sum properties for metric-dependent dimension functions . .

Asit Baran Raha, On completely Hausdorff-completion of a completely

Hausdorff space.

M. Rajagopalan and Bertram Manuel Schreiber, Ergodic automorphisms and affine transformations of locally compact groups..........

N. V. Rao and Ashoke Kumar Roy, Linear isometries of some function

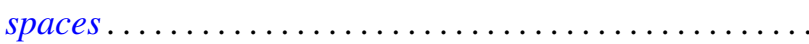

William Francis Reynolds, Blocks and F-class algebras of finite groups

Richard Rochberg, Which linear maps of the disk algebra are multiplicative ...

Gary Sampson, Sharp estimates of convolution transforms in terms of decreasing

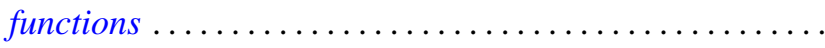

Stephen Scheinberg, Fatou's lemma in normed linear spaces

Ken Shaw, Whittaker constants for entire functions of several complex

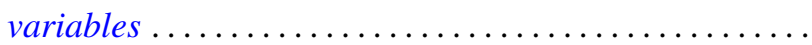

James DeWitt Stein, Two uniform boundedness theorems................ 251

$\mathrm{Li} \mathrm{Pi} \mathrm{Su,} \mathrm{Homomorphisms} \mathrm{of} \mathrm{near-rings} \mathrm{of} \mathrm{continuous} \mathrm{functions} \mathrm{.} \mathrm{.............} 261$

Stephen Willard, Functionally compact spaces, $C$-compact spaces and mappings of minimal Hausdorff spaces....................... 